\title{
On the existence of a superlight scalar boson
}

\author{
Eef van Beveren ${ }^{1, a}$, Susana Coito ${ }^{2, b}$, and George Rupp $2, c$ \\ ${ }^{1}$ Centro de Física Computacional, Departamento de Física, Universidade de Coimbra, P-3004-516 Coimbra, \\ Portugal \\ ${ }^{2}$ Centro de Física das Interacções Fundamentais, Instituto Superior Técnico, Universidade de Lisboa, P- \\ 1049-001 Lisboa, Portugal
}

\begin{abstract}
In this lecture we show that the study of hadronic resonances is severely hampered by the lack of accurate data and, moreover, that for similar reason the study of Weak substructure does not make sufficient progress. We furthermore report on an unexplained high statistics signal that may indicate the existence of a superlight scalar boson.
\end{abstract}

\section{Introduction}

Half a century since the quark model was introduced by Zweig [1] and Gell-Mann [2], a decade later it was followed by the Quantum Chromodynamics (QCD) proposal of Fritzsch, Gell-Mann and Leutwyler [3]. Several discoveries led to a deeper understanding of hadronic interactions and their building blocks [4-12]. Nevertheless, we are still in the dark on the structure of hadronic spectra, which are supposed to be a consequence of the interactions between quarks/antiquarks and glue. We basically live in an era of a plethora of suggestions for the interpretation of hadronic scattering and/or production data. But, as we will discuss in this work, the lack of accurate data does not leave us sufficient hints for a clear direction within the proliferation of models for mesonic and baryonic resonances.

Lattice QCD (LQCD) [13], which was suggested by Wilson shortly after QCD had been proposed and since then has been explored by many researchers, seemed to be the most powerful tool to link theory with experiment. However, four decades later, it is recognised that its application to hadronic production data still needs a much higher degree of perfection than already achieved, leaving enough room for more modest strategies.

In the present work we discuss one of those latter strategies, namely the Resonance-Spectrum Expansion (RSE) [14] for the description of mesonic spectra. The RSE facilitates non-perturbative calculus for scattering and production cross sections and, furthermore, to find resonance pole positions of the scattering amplitude in the complex invariant-mass plane. Here, we will restrict ourselves to the Harmonic-Oscillator version of the RSE (HORSE), since interhadronic dynamics, governed by the glue, is, as we will see in the following, well represented by harmonic-oscillator confinement whereby the oscillator frequency $\omega$ is taken equal to $\omega=190 \mathrm{MeV}$, independent of the meson's

\footnotetext{
ae-mail: eef@teor.fis.uc.pt

${ }^{b}$ Present address: Institute of Modern Physics, CAS, Lanzhou 730000, China; e-mail: susana@impcas.ac.cn

ce-mail: george@ist.utl.pt
} 
flavour content [15]. It is assumed in the RSE that mesons couple to pairs of mesons and vice versa by the creation/annihilation of $q \bar{q}$ pairs. The intensity of pair creation/annihilation is in RSE represented by a parameter $\lambda$ which in principle has to be adjusted to experiment, but which in practice has been found to be rather independent of the meson's flavour content [16].

In Fig. 1 we show the results of HORSE for the charmonium vector states. One observes that

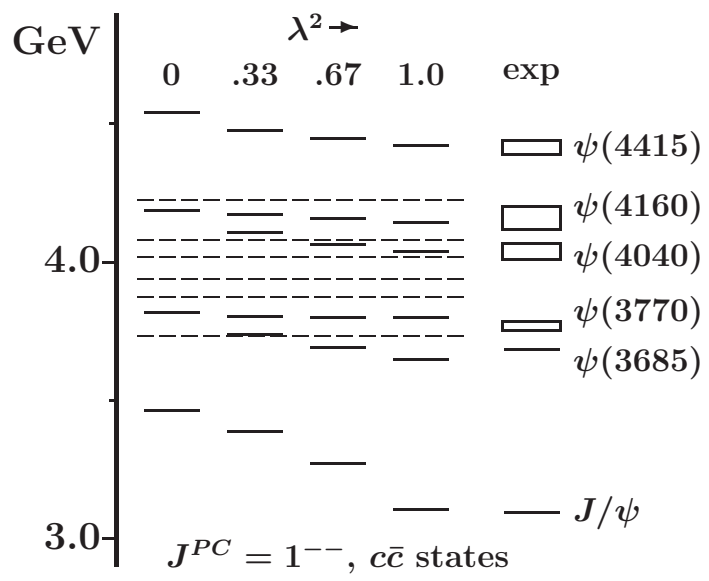

Figure 1. The theoretical values [15] of the central resonance positions for $J^{P C}=1^{--}$charmonium $S$ and $D$ states for various values of the model parameter $\lambda$, compared to the experimental situation [4-10]. The shortdashed lines indicate the threshold positions of the open-charm decay channels, respectively, from the lowest to the highest threshold, DD, $\mathrm{DD}^{*}, \mathrm{D}_{s} \mathrm{D}_{s}, \mathrm{D}^{*} \mathrm{D}^{*}, \mathrm{D}_{s} \mathrm{D}_{s}^{*}$ and $\mathrm{D}_{s}^{*} \mathrm{D}_{s}^{*}$. The long dashes below $\lambda^{2}=0$ represent the masses of the $c \bar{c}$ harmonic-oscillator spectrum, which are degenerate for $S$ and $D$ states except for the ground state at $3.47 \mathrm{GeV}$. The long dashes below non-zero values of $\lambda^{2}$ indicate the real parts of the resonance poles. The latter do not necessarily coincide with central resonance positions for resonances above the DD threshold. Note that the model treats on the same footing bound states below and resonances above the DD threshold.

the system of interacting quarkonium and meson-meson channels explains well the mass splittings between charmonium $S$ and $D$ states. It implies, moreover, that pure $S$ and $D$ states do not exist in nature. We verified that the dominantly $S$ states end up at lower masses than the dominantly $D$ states. In fact, the dominantly $D$ states are such admixtures of $S$ and $D$ states that they almost decouple completely from the meson-meson decay channels, hence form narrow resonances which, due to small mass shifts, do not deviate much from the $c \bar{c}$ harmonic-oscillator spectrum.

It is well known that compositeness may be studied from the appearance of resonance enhancements in the event distributions of scattering and production experiments. An extensive study on hadronic compositeness published by Godfrey and Isgur in Ref. [17] gave us a good insight into the spectrum of quarkonia obtained by the scattering of mesons and by the event distributions of two or more hadrons produced in production experiments.

Less well known is that the phenomenon of dynamically generated resonances, like the light scalar mesons $f_{0}(500), K_{0}^{*}(800), f_{0}(980)$ and $a_{0}(980)$, is related to the main source for the coupling of quarkantiquark systems to systems of two or more hadrons, namely quark-pair creation and annihilation, hence a consequence of hadronic compositeness. Dynamically generated resonances do not make part of the quarkonia spectrum. Different configurations, like multi-quark states [18] or meson molecules [19], have been proposed. Such configurations, however, form an integral part of a complete descrip- 
tion of scattering and production processes based on the coupling, through quark-pair creation, of quarkonia to multi-quark and multi-hadron systems. It was found that the main components which are necessary for a good description of mesonic scattering and production data are quarkonia and two-meson channels [20].

Recent studies in unquenched LQCD confirm the results of much older coupled-channel analyses, such as a calculation [21] of the $D_{s 0}^{*}(2317)$ meson as a scalar $c \bar{s}$ state with an important $D K$ component [22]. However, limitations due to problems in dealing with very broad and overlapping resonances, as well as the physical pion mass, have not yet allowed unquenched LQCD to identify the $K_{0}^{*}(800)$ resonance [23], predicted long ago in the coupled-channel model of Ref. [20]. In Fig. 2 we show the results of HORSE for $S$-wave $K \pi$ scattering cross sections in the isospin $I=1 / 2$ channel [20]. For

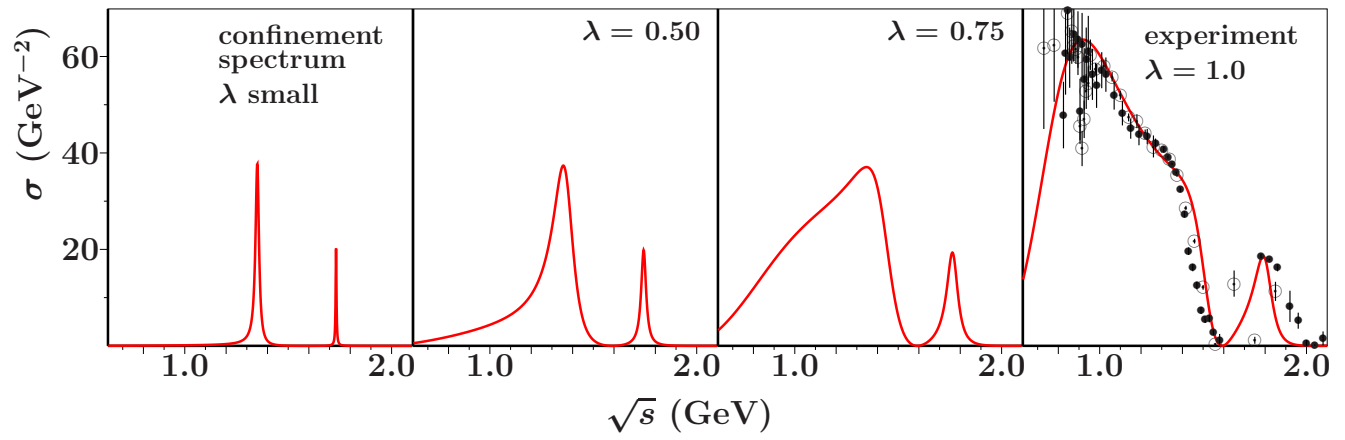

Figure 2. Theoretical [20] and experimental $S$-wave $K \pi$ scattering cross sections in the isospin $I=1 / 2$ channel

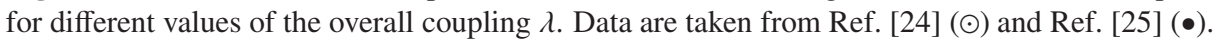

small values of the overall coupling constant $\lambda$, one finds in the model narrow peaks at the harmonicoscillator masses of $u \bar{s}$ states. However, for the model value $\lambda=1$ one obtains a fair agreement between model [20] and experiment [24,25]. Inspection of the model's transition amplitude reveals an additional resonance pole at $s=(0.772-0.281 i)^{2}$, besides the poles that correspond to the harmonicoscillator states. The former resonance pole is absent for small $\lambda$, hence dynamically generated by the interaction between the quark-antiquark and two-meson configurations. At $s=(2.04-0.15 i)^{2}$ we find a further dynamically generated resonance pole, which is unexpected since both data and model show a minimum in the $S$-wave $K \pi$ scattering cross sections in the isospin $I=1 / 2$ channel at the corresponding invariant mass. The latter phenomenon underlines once more the need for highstatistics data.

It is basically unknown that a further consequence of hadronic compositeness is the appearance of non-resonant threshold enhancements. A theoretical model for threshold enhancements in hadronic production amplitudes, based on quark-antiquark pair creation, was formulated in Ref. [26] and further developed in Refs. [27, 28]. This model shows that one must expect non-resonant enhancements in the amplitudes just above pair-creation thresholds, which, in the case of stable hadrons, are accompanied by a clear minimum at threshold, as observed in experiment for the process $e^{+} e^{-} \rightarrow b \bar{b}$, measured and analysed by the BaBar Collaboration [29]. As also remarked by BaBar in their paper, the large statistics and the small energy steps of the scan make it possible to clearly observe the dips at the opening of the thresholds corresponding to the $B \bar{B}^{*}+\bar{B} B^{*}$ and $B^{*} \bar{B}^{*}$ channels. However, experimental evidence of this phenomenon is scarce, since it needs event counts with high statistics and good 
resolution. Nevertheless, in some cases signals, albeit often feeble, can be seen in experimental data for hadronic production [30].

In Sect. 2 we study some examples of possibly observed threshold enhancements, thereby stressing the need for more accurate data. The possibly observed superlight boson is discussed in Sect. 3 .

\section{Threshold enhancements}

In Ref. [26] the generic relation

$$
P=\mathfrak{J} m(Z)+T Z
$$

between two-particle scattering $(T)$ and production $(P)$ amplitudes is studied in a microscopic multichannel model for meson-meson scattering with coupling to confined quark-antiquark channels. The amplitude $T$ in expression (1) is supposed to contain the resonance poles which occur in scattering, whereas $Z$ is a smooth function of invariant mass. Threshold enhancements occur in production amplitudes as a consequence of the shape of $\mathfrak{J} m(Z)$, which in the ideal case of no further nearby thresholds rises sharply just above threshold. For larger invariant masses $\mathfrak{J} m(Z)$ first reaches a maximum and then falls off exponentially. As a consequence, production amplitudes show non-resonant yet resonant-like enhancements just above threshold.

\subsection{Electron-positron annihilation into open-bottom pairs}

In Fig. 3 we show data on hadron production in electron-positron annihilation, published by the BaBar Collaboration in Ref. [29]. In Fig. 3a we show the full data in the mass region contained between the

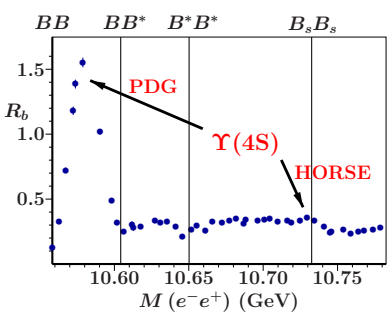

(a)

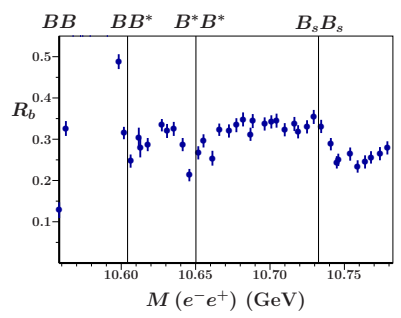

(b)

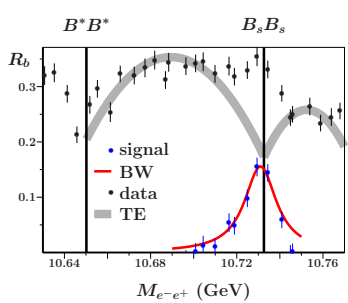

(c)

Figure 3. $R_{b}$ as measured by $\mathrm{BaBaR}[29]$ in electron-positron annihilation; (a): Full data, (b): without the enhancement at $10.58 \mathrm{GeV}$, (c): near the $B_{s} B_{s}$ threshold. In (c) a Breit-Wigner (BW) approximation is shown for the difference signal between the data and threshold-enhancement (TE) structures.

$B B$ and $B B^{*}$ threshold, whereas in Fig. $3 \mathrm{~b}$ we concentrate on the data above the $B B^{*}$ threshold. We observe in Fig. 3 three threshold enhancements at the opening of $B B, B B^{*}$ and $B^{*} B^{*}$, which most likely are non-resonant. The BaBar Collaboration remarks in Ref. [29] that large statistics and the small energy steps of the scan make it possible to clearly observe dips at the opening of the thresholds corresponding to $B B^{*}$ and $B^{*} B^{*}$. The shapes of the various threshold enhancements do not show the just mentioned exponential tails, as a result of interference between the various configurations $B B$, $B B^{*}, B^{*} B^{*}$, and so on. Such configurations are of course also present below threshold, though not capable of materialising. 
In Fig. 3c we show the difference signal near the opening of $B_{s} B_{s}$, which results from subtracting the $B^{*} B^{*}$ and $B_{s} B_{s}$ threshold enhancements from the full data. A Breit-Wigner approximation to the difference signal clearly shows the shape of a resonance. According to us, this is the $\Upsilon(4 S)$ state, rather than the large enhancement at $10.58 \mathrm{GeV}$ and also in fair agreement with the predictions in Refs. $[15,16]$.

The reason why the $B B$ threshold enhancement is much more pronounced than those for the $B B^{*}$ and $B^{*} B^{*}$ channels can be explained by the presence of a strong resonance pole in the scattering amplitude on the real invariant-mass axis just below the $B B$ threshold at about $10.5 \mathrm{GeV}$. Data on $e^{+} e^{-} \rightarrow \pi^{+} \pi^{-} \Upsilon(2 S, 1 S) \rightarrow \pi^{+} \pi^{-} e^{+} e^{-}$published by the BaBar Collaboration [31] are further analysed in Ref. [32]. In Fig. 4 we depict the signal at $10495 \pm 5 \mathrm{MeV}$, which was obtained in Ref. [32] for the $\Upsilon\left(2{ }^{3} D_{1}\right)$ resonance and which is in good agreement with the prediction in Ref. [15]. The signal,

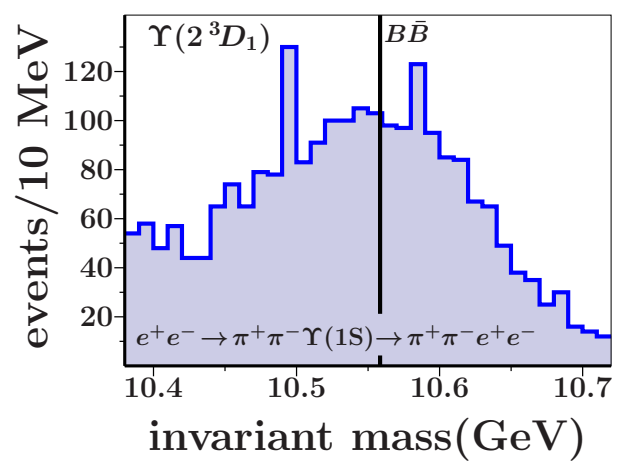

Figure 4. Narrow signal obtained for the $\Upsilon\left(2{ }^{3} D_{1}\right)$ resonance at $10495 \pm 5 \mathrm{MeV}$ [32]. Data are taken from Ref. [31]. For completeness, the $B \bar{B}$ threshold is indicated.

which is even more prominent than the enhancement at $10.58 \mathrm{GeV}$, implies a strong resonance pole on the real axis in the complex invariant-mass plane. It is this pole that mainly contributes to the threshold enhancement above the $B \bar{B}$ threshold.

\subsection{Electron-positron annihilation into open-charm pairs}

Further threshold enhancements are discussed in Ref. [30]. Here we will concentrate on the reaction of $e^{+} e^{-}$annihilation into open-charm pairs, which can be observed at and above the $D \bar{D}$ threshold. We assume here that the reaction takes place via a photon and the $c \bar{c}$ propagator, through the creation of a light $q \bar{q}$ pair. However, many competing configurations may be formed, increasing in number for higher invariant masses. Furthermore, it seems we may conclude from experiment that stable opencharm hadrons have more probability to be formed near threshold, where kinetic energy is almost zero. Hence, if it were not for phase space and the centrifugal barrier, $D \bar{D}$ pairs would be produced most likely just above threshold.

For total invariant mass below but close to the $D D^{*}$ threshold, we assume that the probability of $D \bar{D}$ creation is already reduced because of the non-vanishing probability of creating a virtual $D D^{*}$ pair. Just above the $D D^{*}$ threshold, $D \bar{D}$ creation decreases rapidly. The expected corresponding nonresonant contribution of the production amplitude should thus exhibit this feature. In this respect, an important observation was published by the BES Collaboration in Ref. [33]. To our knowledge, the BES Collaboration was the first to discover that the $\psi(3770)$ cross section is built up by two different amplitudes, viz. a relatively broad signal and a rather narrow $c \bar{c}$ resonance. For the narrow resonance, which probably corresponds to the well-established $\psi(1 D)(3770)$, the BES Collaboration measured 
a central resonance position of $3781.0 \pm 1.3 \pm 0.5 \mathrm{MeV}$ and a width of $19.3 \pm 3.1 \pm 0.1 \mathrm{MeV}$ (their solution 2). If the latter parameters are indeed confirmed, it would be yet another observation of a quark-antiquark resonance width that is very different from the world average $(87.04 \pm 0.33 \mathrm{MeV}$ [34] in this case), after a similar result was obtained by the BaBar Collaboration in Ref. [29], for $b \bar{b}$ resonances. Concerning the broader structure, the BES Collaboration indicated, for their solution 2, a central resonance position of $3762.6 \pm 11.8 \pm 0.5 \mathrm{MeV}$ and a width of $49.9 \pm 32.1 \pm 0.1 \mathrm{MeV}$. The signal significance for the new enhancement is $7.6 \sigma$ (solution 2). It was explained as a possible diresonance [35] or heavy molecular state [36]. Here, we assume that the broader structure is most likely caused by the non-resonant contribution to the production amplitude given in Eq. (1), thus lending further support to the idea that the $\psi(1 D)(3770)$ enhancement consists of two distinct signals.

The data shown in Fig. 5 are fitted by assuming

$$
Z=\mathcal{N} p r_{0} \exp \left\{-\left(p r_{0}\right)^{2}\right\} e^{i \phi} \quad\left(4 p^{2}=s-4 M_{D}^{2}\right)
$$

for the $Z$ function in Eq. (1), and

$$
T=\frac{\Gamma_{\psi} / 2}{\sqrt{s}-M_{\psi}+i \Gamma_{\psi} / 2}
$$

for the scattering amplitude in Eq. (1). The theoretical curve in Fig. 5 corresponds to $M_{\psi}=3782$ $\mathrm{MeV}, r_{0}=0.36 \mathrm{fm}, \Gamma_{\psi}=18 \mathrm{MeV}$ and $\phi=0.7 \pi$. The overall normalisation factor $\mathcal{N}$ in Eq. (2) is adjusted to the data.

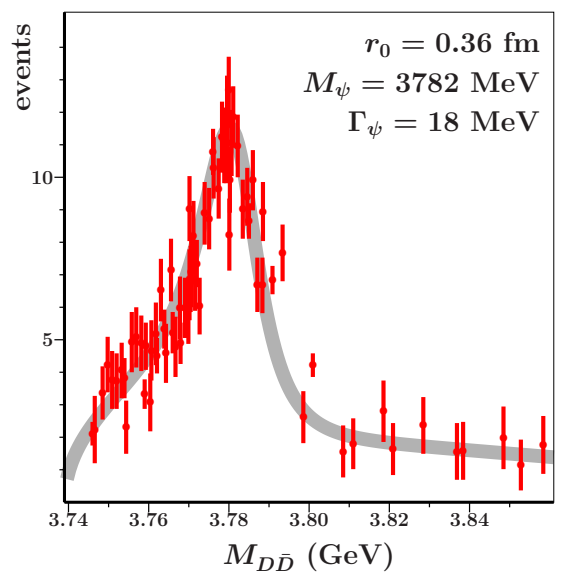

Figure 5. Comparison of experimental data obtained by the BES Collaboration [33] and the cross section resulting from Eq. (1), by the use of expressions (2) and (3). The non-resonant contribution dominates for larger relative $D \bar{D}$ momentum $p$. The $\mathrm{BW}$ parameters of the $c \bar{c}$ resonance are $M(\psi(1 D))=3.782 \mathrm{GeV}$ and $\Gamma(\psi(1 D))=18 \mathrm{MeV}$.

Note the sharp interference dip at about $3.81 \mathrm{GeV}$, which can also be observed in data published by the BaBar Collaboration [37], but with even less statistics.

\section{$2.3 D^{0} K^{-}$invariant-mass distribution}

When the $D_{s J}^{*}(2860)^{+}$meson was discovered by the BaBar Collaboration [38], only the $D K$ decay mode was detected, which made an assignment as a radially excited scalar $c \bar{s}$ meson plausible [3941], though other configurations such as a $3^{-}$state [41-43] could not be excluded. For a discussion of additional options, see Ref. [44]. The later observed $D^{*} K$ mode [45] at first seemed to exclude the $0^{+}$scenario for the $D_{s J}^{*}(2860)^{+}$resonance. But the true situation may be subtler, involving two overlapping resonances, viz. one scalar $2{ }^{3} P_{0}$ and one tensor $2{ }^{3} P_{2}$ charm-strange meson [46]. However, 
in a recent study the LHCb Collaboration finds overlapping spin- 1 and spin-3 resonances at a mass $2.86 \mathrm{GeV}$, by analysing the resonant $D^{0} K^{-}$substructure of $B_{s}^{0} \rightarrow \bar{D}^{0} K^{-} \pi^{+}$decays with the technique of Dalitz-plot analysis [47, 48]. Here we will study the latter result.

The angular analysis of the $D_{s J}^{*}(2860)^{-} \rightarrow D^{0} K^{-}$decays supports natural parity for the resonance, i.e., $J^{P}=0^{+}, 1^{-}, 2^{+}, 3^{-}, \ldots$ Its mass spectrum for harmonic-oscillator confinement [16] is given in Table 1. We observe at a glance that, besides the well-established ${ }^{3} S_{1}(2110)$ and ${ }^{3} P_{2}(2572)$

\begin{tabular}{||c||c||}
\hline \hline Mass $(\mathrm{GeV})$ & States \\
\hline 3.305 & $3^{3} P_{0} 3^{3} P_{2} 2^{3} F_{2} 2^{3} F_{4} 1^{3} H_{4} 1^{3} H_{6}$ \\
3.115 & $\mathbf{3}^{3} \boldsymbol{S}_{\mathbf{1}} 2^{3} D_{1} 2^{3} D_{3} 1^{3} G_{3} 1^{3} G_{5}$ \\
2.925 & $\mathbf{2}^{\mathbf{3}} \boldsymbol{P}_{\mathbf{0}} \mathbf{2}^{\mathbf{3}} \boldsymbol{P}_{\mathbf{2}} \mathbf{1}^{\mathbf{3}} \boldsymbol{F}_{\mathbf{2}} \mathbf{1}^{\mathbf{3}} \boldsymbol{F}_{\mathbf{4}}$ \\
2.735 & $2^{3} S_{1} 1^{3} D_{1}(2709) 1^{3} D_{3}$ \\
2.545 & $1^{3} P_{0} 1^{3} P_{2}(2572)$ \\
2.355 & $1^{3} S_{1}(2110)$ \\
\hline \hline
\end{tabular}

Table 1. $D_{s}$ natural-parity HO states and their masses. When experimental central resonance masses are measured, those are shown in brackets. The states in boldface are discussed in the text.

resonances, most of the predicted states have not even been observed or their quantum numbers are still to be determined. In Ref. [49] the assignment $2^{3} S_{1}$ for the resonance at $2.71 \mathrm{GeV}$ is disputed. As can be read from Table 1, we expect four resonances in the mass interval $2.735-2.925 \mathrm{GeV}$, namely $2^{3} P_{0}, 2^{3} P_{2}, 1^{3} F_{2}$ and $1^{3} F_{4}$, while out of the five predicted resonances in the mass interval 2.925-3.115 $\mathrm{GeV}$ we expect for $3^{3} S_{1}$ the larger mass shift and thus to come out closer to $2.925 \mathrm{GeV}$ than the other four.

In Fig. 6 we show the $D^{0} K^{-}$mass distribution in the mass interval $2.75-3.0 \mathrm{GeV}$ as published by $\mathrm{LHCb}[47,48]$. Besides an estimate for a possible background, the threshold enhancements at

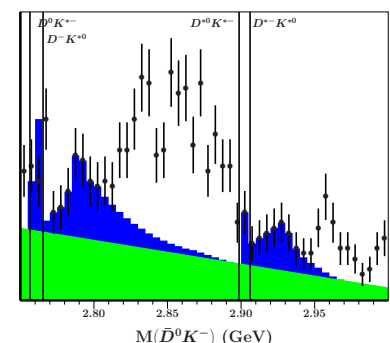

(a)

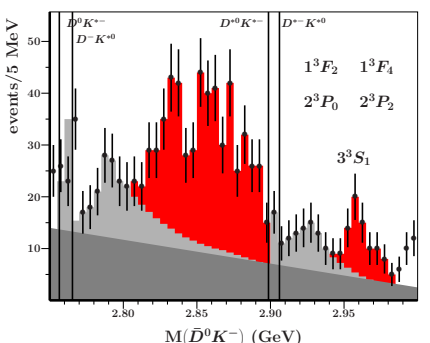

(b)

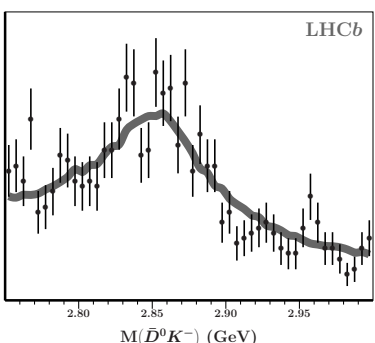

(c)

Figure 6. $D^{0} K^{-}$mass distribution in $B_{s}^{0} \rightarrow \bar{D}^{0} K^{-} \pi^{+}$decay [47, 48]; (a): Our suggestion for background and threshold enhancements, (b): Highlighting the remaining structures, (c): LHCb representation of the data.

the openings of the $D^{0} K^{*-} / D^{-} K^{* 0}$ channels and the $D^{* 0} K^{*-} / D^{*-} K^{* 0}$ channels are shown in Fig. 6a. The remaining signal is highlighted in Fig. $6 \mathrm{~b}$. The $3{ }^{3} S_{1}$ assignment for the peak at $2.96 \mathrm{GeV}$ seems to be in good agreement with our expectation from the $\mathrm{HO}$ value of $3115 \mathrm{MeV}$ and a mass shift of 
roughly $160 \mathrm{MeV}$ due to meson loops. The signal peaking at about $2.86 \mathrm{GeV}$ could consist of 2,3 or 4 overlapping resonances. This issue can only be solved with much better statistics and bin sizes that do not exceed 1.0 MeV. The LHCb solution for the data shown here is depicted in Fig. 6c.

\subsection{Weak substructure?}

This section is devoted to the recently observed (pseudo)scalar, most probably scalar [50, 51], enhancement in the 120-135 GeV interval $[52,53]$ in the light of non-resonant threshold enhancements.

In Ref. [54] the CMS Collaboration collected diphoton events in proton-proton collisions corresponding to integrated luminosities of $5.1 \mathrm{fb}^{-1}$ at a centre-of-mass energy of $7 \mathrm{TeV}$, and $19.7 \mathrm{fb}^{-1}$ at $8 \mathrm{TeV}$. The ATLAS Collaboration published similar results [55] based on data samples corresponding to integrated luminosities of up to $20.7 \mathrm{fb}^{-1}$ at $8 \mathrm{TeV}$, and $4.6 \mathrm{fb}^{-1}$ at $7 \mathrm{TeV}$. Those data are collected in Fig. 7a.

Besides the bump at about $125 \mathrm{GeV}$, one observes a clear dip in the diphoton invariant-mass distribution at about $115 \mathrm{GeV}$. Its signal is rather similar to the threshold dips observed by the BaBar Collaboration [29] and which are depicted in Fig. 3. Hence, could there exist a threshold at $115 \mathrm{GeV}$ for particle-antiparticle production? If so, the masses of the particles should be about $57.5 \mathrm{GeV}$ each.

Composite heavy gauge bosons and their spin-zero partners, the latter with a mass in the range 50-60 GeV, were considered long ago [56] and studied in numerous works (see e.g. Refs. [57-62]). To date, no experimental evidence of their existence has been reported. More recently the interest in Weak substructure has revived [63-67].

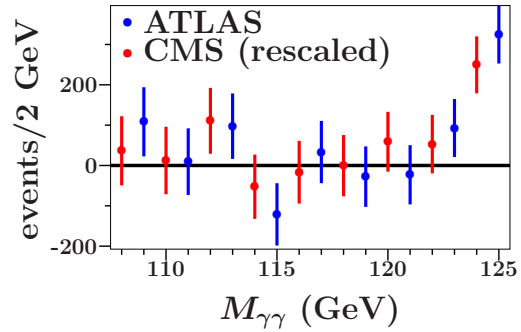

(a)

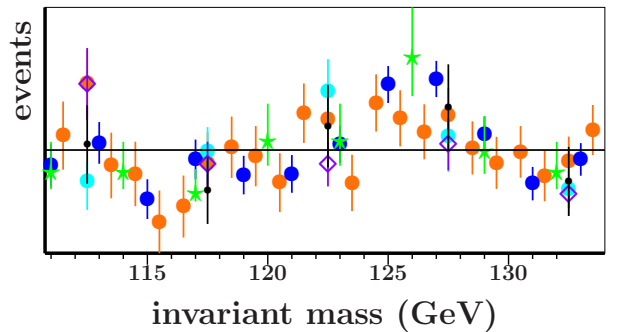

(b)

Figure 7. (a): Diphoton signals after background subtraction, published by the CMS Collaboration [54] (•) and

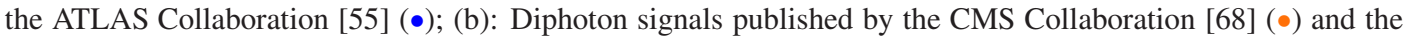
ATLAS Collaboration [55] (•), four-lepton signals published by the CMS Collaboration [69] ( $\star$ ) and the ATLAS Collaboration [55] (•), invariant-mass distributions for $\tau \tau$ in $e^{+} e^{-} \rightarrow \tau \tau(\gamma)(\diamond)$ and $\mu \mu$ in $e^{+} e^{-} \rightarrow \mu \mu(\gamma)(\bullet)$ published by the L3 Collaboration [70].

In Fig. 7b we collect invariant-mass distributions for a variety of two-particle production processes, namely diphoton signals after background subtraction published by the CMS [68] and ATLAS Collaborations [55], four-lepton signals published by the CMS [69] and ATLAS Collaborations [55], and for $\tau \tau$ in $e^{+} e^{-} \rightarrow \tau \tau(\gamma)$ and $\mu \mu$ in $e^{+} e^{-} \rightarrow \mu \mu(\gamma)$ published by the L3 Collaboration in Ref. [70]. The data shown in Fig. 7 indeed seem to suggest that the total signal in the 115-133 GeV energy interval is built up by two different amplitudes, viz. a broad non-resonant signal and a resonance at about $125 \mathrm{GeV}$. 
In order to proceed let us assume that a (pseudo-)scalar partner of the $Z$-boson exists, say a $\tilde{Z}(57)$. By its quantum numbers we may expect that the $\tilde{Z}(57)$ does not show up in dilepton cross sections, or at least not as a very clear resonance. But it could be visible in diphoton data. Indeed, a promising analysis of the L3 Collaboration [71] does show that $Z(91) \rightarrow \gamma \tilde{Z}(57)$ transitions cannot be excluded a priori. In their analysis, the L3 Collaboration searched for anomalous $Z \rightarrow \gamma \gamma \gamma$ events with the L3 detector at LEP and concluded that no significant deviations were observed from the $e^{+} e^{-} \rightarrow \gamma \gamma \gamma$ events expected by QED. We do not contest here the conclusion of the L3 Collaboration, since with a sample of 87 candidate events, against $(76.3 \pm 2.8)$ events expected from QED processes, one can hardly expect sufficient resolution and statistics for evidence of the existence of a hard-to-be-detected pseudoscalar partner of the $Z$ boson. Nevertheless, it is justified to pay here closer attention to the result obtained by the L3 Collaboration.

In Fig.8a/b we show the L3 data for the three one-photon CM energies for each of the candidate events. The L3 Collaboration collected the one-photon CM energies as a function of $M_{\gamma} / \sqrt{s}$. Here, we have converted that information into $M_{\gamma}$, thereby assuming $\sqrt{s}=M_{Z}$. With a green band we indicate where we expect the photons from the radiative process $Z(91) \rightarrow \gamma \tilde{Z}(57)$. We observe that
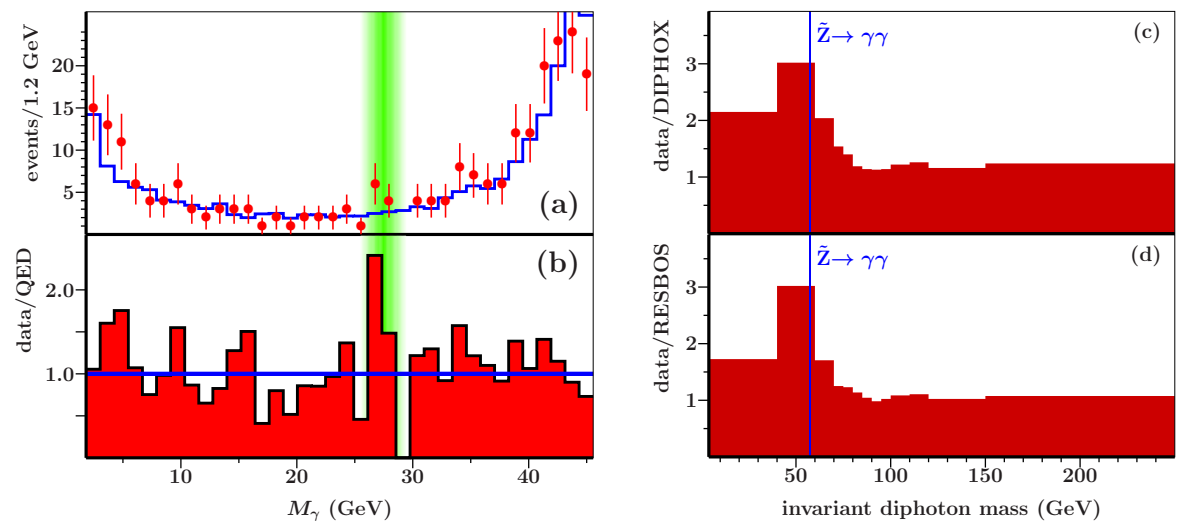

Figure 8. (a): Experimental data for the three one-photon CM energies of the candidate $Z \rightarrow \gamma \gamma \gamma$ events obtained by the L3 Collaboration [71], assuming $\sqrt{s}=M_{Z}$. The histogram was obtained by the L3 Collaboration from a Monte-Carlo simulation for the expected number of events predicted by QED. With the green band we indicate where we expect photons from the radiative process $Z(91) \rightarrow \gamma \tilde{Z}(57)$ for the case that $\tilde{Z}(57)$ has a mass of 57.5 GeV. (b): The same data as shown in (a), but now measured events divided by QED expected events. (c, d): Measured over expected events for diphoton invariant-mass distributions published by the CMS Collaboration [72], for (c) DIPHOX and (d) RESBOS.

most of the data agree well with the expectation from QED. Nevertheless, be it a coincidence or not, in the mass region where we expect a signal from $Z(91) \rightarrow \gamma \tilde{Z}(57)$ events, we observe a small enhancement. The latter can be better demonstrated by showing the ratio of measured signal over QED prediction, as also depicted in Fig. 8b. Here one clearly observes a modest enhancement for exactly the expected $\tilde{Z}(57)$ mass of $57.5 \mathrm{GeV}$.

In diphoton invariant-mass distributions published by the CMS Collaboration [72], shown in Fig. 8c for predictions of a DIPHOX data simulator and in Fig. 8d for predictions of RESBOS, one also observes an excess of three times more observed events than predicted in the 40-60 GeV mass interval. This could be in agreement with diphotons stemming from the decay process $\tilde{Z}(57) \rightarrow \gamma \gamma$. 
We have thus shown here that the described threshold phenomena, observed for mesons, may also have an application to heavy gauge bosons at high energies, where the weak interactions are in fact not weak anymore. Confirmation of such effects would indicate compositeness in this sector of the Standard Model, too. Moreover, it could offer an explanation for the enhancement around $125 \mathrm{GeV}$ seen by ATLAS and CMS at LHC, as a possible alternative to a generally accepted Higgs-like particle of such a mass. A consequence of this scenario would be the existence of gauge-boson partners, of lower mass and with different quantum numbers, being either scalars or pseudoscalars. The LHC and LEP data we have presented above indeed hint at the existence of such partners, namely a $\tilde{Z}(57)$ at 57.5 GeV. Moreover, there are also indications [73], albeit feeble, of $Z$-like recurrences, viz. at about 210 and $240 \mathrm{GeV}$. However, only much improved statistics and resolution can settle the issue of a possible weak substructure and its manifestation through new heavy bosons.

\section{Existence of a superlight scalar boson}

In Ref. [74] a variety of indications were presented of the possible existence of a light boson with a mass of about $38 \mathrm{MeV}$, henceforth referred to as $E(38)$. These indications amounted to a series of low-statistics observations all pointing in the same direction, and one high-statistics observation, which might be interpreted as the discovery of the $E(38)$.

\subsection{Motivation}

In Ref. [75] an $S O(4,2)$ conformally symmetric model was proposed for strong interactions at low energies, based on the observation [76-78] that confinement can be described by an anti-De Sitter (aDS) background geometry. The possibility of such a strategy had already been studied, almost a century ago, by H. Weyl [79], who found that the dynamical equations of gauge theories retain their flat-space-time form when subject to a conformally-flat metrical field instead of the usual Minkowski background. The unification of electromagnetism and strong interactions can be justified by the very subtle balance between these forces in the nucleus, where just one neutron more or less can make the difference between stability or instability.

Confinement of quarks and gluons was in Ref. [75] modelled by the introduction of two scalar fields, which spontaneously break the $S O(4,2)$ symmetry down to $S O(3,2)$ and $S O(3) \otimes S O(2)$ symmetry, respectively. Moreover, a symmetric second-order tensor field was defined that serves as the metric for flat space-time, coupling to electromagnetism. Quarks and gluons, which to lowest order do not couple to this tensor field, are confined to an aDS universe [80], having a finite radius in the flat space-time. This way, the model describes quarks and gluons, which oscillate with a universal frequency — independent of the flavour mass — inside a closed universe, as well as photons, which freely travel through flat space-time.

The fields in the model of Ref. [75] comprise one real scalar field $\sigma$ and one complex scalar field $\lambda$. Their dynamical equations were solved in Ref. [75] for the case that the respective vacuum expectation values, given by $\sigma_{0}$ and $\lambda_{0}$, satisfy the relation

$$
\left|\sigma_{0}\right| \gg\left|\lambda_{0}\right|
$$

A solution for $\sigma_{0}$ of particular interest leads to aDS confinement, via the associated conformally flat metric given by $\sigma \eta_{\mu \nu}$. Furthermore, the only quadratic term in the Lagrangian of Ref. [75] is proportional to $-\sigma^{2} \lambda^{*} \lambda$. Hence, under the condition of relation (4), one obtains, after choosing vacuum expectation values, a light $\sigma$ field associated with confinement, and a very heavy complex $\lambda$ field 
associated with electromagnetism. Here, we will study the — supposedly light — mass of the scalar field that gives rise to confinement.

The conformally symmetric model of Ref. [75] in itself does not easily allow for interactions between hadrons, as each hadron is described by a closed universe. Therefore, in order to compare the properties of this model to the actually measured cross sections and branching ratios, the model has been further simplified, such that only its main property survives, namely its flavour-independent oscillations. This way the full aDS spectrum is, via light-quark-pair creation, coupled to the channels of two - or more - hadronic decay products for which scattering amplitudes can be measured, thus relating observed resonances to the aDS spectrum.

The aDS spectrum reveals itself through the structures observed in hadronic invariant-mass distributions. However, as we have shown in the past (see Ref. [49] and references therein), there exists no simple relation between enhancements in the experimental cross sections and the aDS spectrum. Nevertheless, this was studied in parallel, for mesons, in a coupled-channel model in which quarks are confined by a flavour-independent harmonic oscillator $[15,16]$. Empirically, based on numerous data on mesonic resonances measured by a large variety of experimental collaborations, it was found [81] that an aDS oscillation frequency of $\omega=190 \mathrm{MeV}$ agrees well with the observed results for mesonmeson scattering and meson-pair production in the light [20], heavy-light [22], and heavy [32, 82] flavour sectors, thus reinforcing the strategy proposed in Ref. [75].

A further ingredient of the model for the description of non-exotic quarkonia, namely the coupling of quark-antiquark components to real and virtual two-meson decay channels [83] via ${ }^{3} P_{0}$ quark-pair creation, gives us a clue about the size of the mass of the $\sigma$ field. For such a coupling it was found that the average radius $r_{0}$ for light-quark-pair creation in quarkonia can be described by a flavourindependent mass scale, given by $M=\frac{1}{2} \omega^{2} \mu r_{0}^{2}$, where $\mu$ is the effective reduced quarkonium mass. In earlier work, the value $\rho_{0}=\sqrt{\mu \omega} r_{0}=0.56[15,16]$ was used, which results in $M=30 \mathrm{MeV}$ for the corresponding mass scale. However, the quarkonium spectrum is not very sensitive to the precise value of the radius $r_{0}$, in contrast with the resonance widths. In more recent work [84, 85], slightly larger transition radii have been applied, corresponding to values around $40 \mathrm{MeV}$ for $M$. Nevertheless, values of $30-40 \mathrm{MeV}$ for the flavour-independent mass $M$ do not seem to bear any relation to an observed quantity for strong interactions. However, in Refs. $[74,86]$ we have presented experimental evidence for the possible existence of a quantum with a mass of about $38 \mathrm{MeV}$, which in light of its relation to the ${ }^{3} P_{0}$ mechanism we suppose to mediate quark-pair creation. Moreover, its scalar properties make it a perfect candidate for the quantum associated with the above-discussed scalar field for confinement.

\subsection{Interference effects}

In Ref. [87] notice was made of an apparent interference effect around the $D_{s}^{*} \bar{D}_{s}^{*}$ threshold in the invariant-mass distribution of $e^{+} e^{-} \rightarrow J / \psi \pi^{+} \pi^{-}$events, which we observed in preliminary radiation data of the BaBar Collaboration [88]. The effect, with a periodicity of about $74 \mathrm{MeV}$, could be due to interference between the typical oscillation frequency of $190 \mathrm{MeV}$ of the $c \bar{c}$ pair, as in the model of Refs. [15, 16], and that of the gluon cloud. Later, in Ref. [89], evidence was reported of small oscillations in electron-positron and proton-antiproton annihilation data, with a periodicity of $76 \pm 2$ $\mathrm{MeV}$, independent of the beam energy. The latter observations are summarised in Fig. 9.

Amongst the various scenarios to explain the phenomenon presented in Ref. [89], one was rather intriguing, namely the postulated existence of gluonic oscillations, possibly surface oscillations, with a frequency of about $38 \mathrm{MeV}$. These would then, upon interfering with the universal quarkonium frequency $\omega=190 \mathrm{MeV}[15,16]$, lead to the observed oscillations. However, here we will show that the phenomenon is most likely to be associated with the interquark exchange of a boson with 

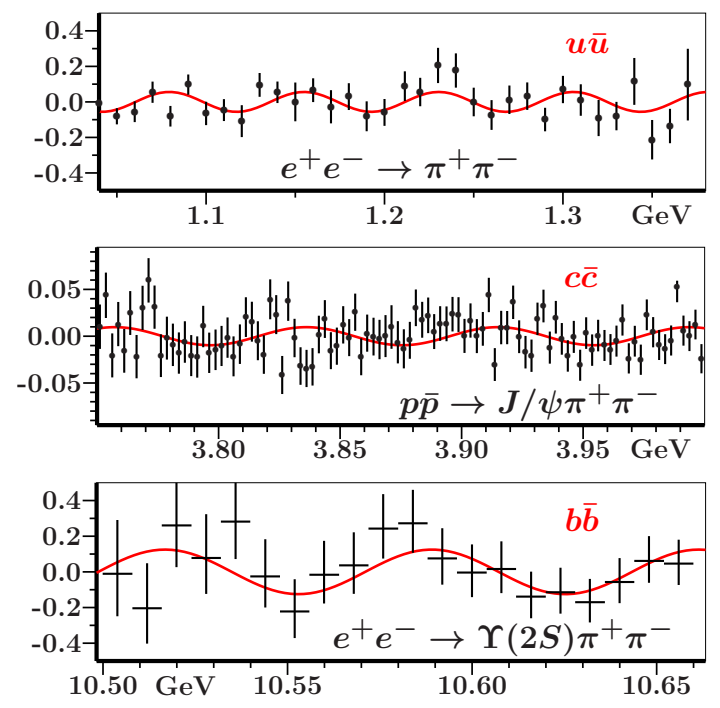

Figure 9. Fits to the residual data, after subtraction of global fits to: $e^{+} e^{-} \rightarrow \pi^{+} \pi^{-}$data of the CMD-2 Collaboration [90], with a period of $78 \pm 2 \mathrm{MeV}$ and an amplitude of $\approx 5 \%$ (top); $p \bar{p} \rightarrow J / \psi \pi^{+} \pi^{-}$data of the CDF Collaboration [91], with a period of $79 \pm 5 \mathrm{MeV}$ and an amplitude of about $0.75 \%$ (middle); $e^{+} e^{-} \rightarrow \Upsilon(2 S) \pi^{+} \pi^{-}$ data of the BaBar Collaboration [31], with a period of $73 \pm 3 \mathrm{MeV}$ and an amplitude of some $12.5 \%$ (bottom).

a mass of about $38 \mathrm{MeV}$. Moreover, from the fact that the observed oscillations are more intense for bottomonium than for light quarks, we assume that the coupling of this light boson to quarks increases with the quark mass. This seems to correspond well to the scalar particle of the model of Ref. [75], and to the enigmatic mass parameter related to the ${ }^{3} P_{0}$ pair-creation mechanism [15].

In Ref. [31], the BaBar Collaboration presented an analysis of data on $e^{+} e^{-} \rightarrow \pi^{+} \pi^{-} \Upsilon\left(1,2{ }^{3} S_{1}\right)$ $\rightarrow \pi^{+} \pi^{-} \ell^{+} \ell^{-}(\ell=e$ and $\ell=\mu)$, with the aim to study hadronic transitions between $b \bar{b}$ excitations and the $\Upsilon\left(1{ }^{3} S_{1}\right)$ and $\Upsilon\left(2{ }^{3} S_{1}\right)$, based on $347.5 \mathrm{fb}^{-1}$ of data taken with the BaBar detector at the PEP-II storage rings. The selection procedure for the data is well described by BaBar in Refs. [31, 92, 93].

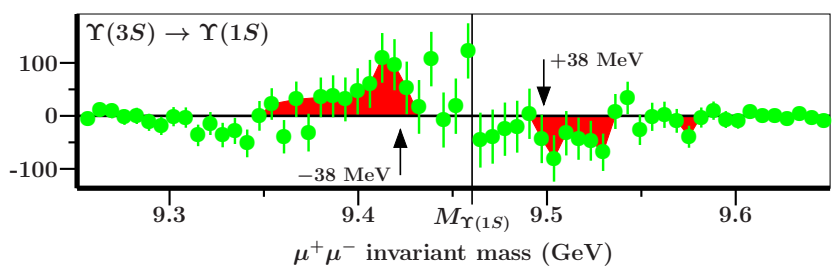

Figure 10. Event distribution of the excess signal taken from Ref. [93], in the invariant- $\mu^{+} \mu^{-}$-mass distribution for the reaction $\Upsilon\left(2{ }^{3} S_{1}\right) \rightarrow \pi^{+} \pi^{-} \Upsilon\left(1{ }^{3} S_{1}\right) \rightarrow \pi^{+} \pi^{-} \mu^{+} \mu^{-}$. Statistical errors are shown by vertical bars. The shaded areas (dark, red in online version) are discussed in the text. The vertical line indicates $M_{\mu^{+} \mu^{-}}=M_{\Upsilon\left(1{ }^{3} S_{1}\right)}$.

A particularly interesting study, published by the BaBar Collaboration [93], is the asymmetry with respect to a Gaussian distribution for the reaction $e^{+} e^{-} \rightarrow \Upsilon\left(2{ }^{3} S_{1}\right) \rightarrow \pi^{+} \pi^{-} \Upsilon\left(1{ }^{3} S_{1}\right) \rightarrow \pi^{+} \pi^{-} \mu^{+} \mu^{-}$. We depict the result in Fig. 10. We observe that, with respect to the Gaussian distribution, there is 
an excess of data for $M_{\mu^{+} \mu^{-}}$below the $\Upsilon\left(1{ }^{3} S_{1}\right)$ mass, and a deficit of data for $M_{\mu^{+} \mu^{-}}$thereabove. Moreover, the analysis in Ref. [93] took all known possible origins of asymmetry into account. Consequently, what is left (see Fig. 10) cannot be explained by known physics. Furthermore, it states that the, here reported, systematic uncertainties due to the differences between data and simulation in the processes $\Upsilon\left(1{ }^{3} S_{1}\right) \rightarrow \tau^{+} \tau^{-}$and $\Upsilon\left(1{ }^{3} S_{1}\right) \rightarrow \mu^{+} \mu^{-}$cancel, at least in part, in their ratio. This implies that a similar excess is found in the $\Upsilon\left(1{ }^{3} S_{1}\right) \rightarrow \tau^{+} \tau^{-}$decay.

In order to explain the structures in the deficit signal, we must assume that the $E(38)$ can be loosely bound inside a $b \bar{b}$ state, giving rise to a kind of hybrid. This was discussed to some detail in Ref. [86]. In Fig. 11, we show the event distribution for the invariant mass $\Delta M$, which is defined [31] by $\Delta M=$ $M_{\pi^{+} \pi^{-} \mu^{+} \mu^{-}}-M_{\mu^{+} \mu^{-}}$, where the latter mass is supposed to be the $\Upsilon\left(1{ }^{3} S_{1}\right)$ mass. Thus, a signal with the

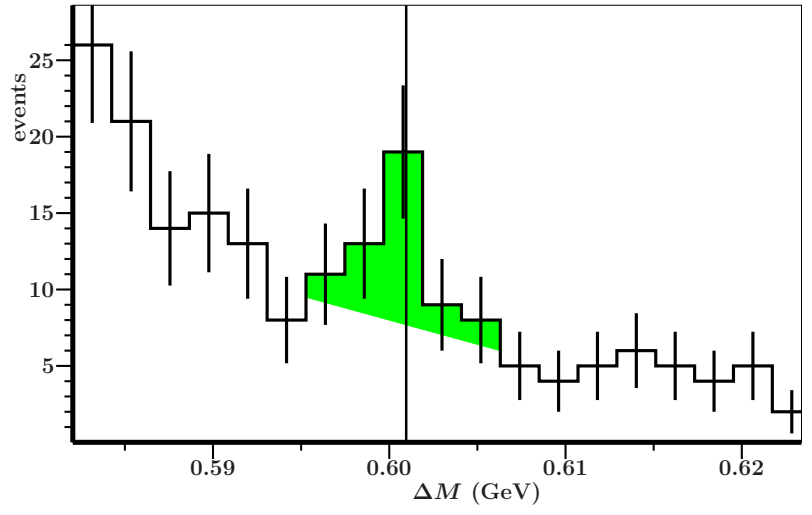

Figure 11. Possible sign of the $\Upsilon^{\prime}\left(2{ }^{3} S_{1}\right)$ hybrid. The vertical line indicates where $M_{\Upsilon\left(2^{3} S_{1}\right)}+38 \mathrm{MeV}$ comes out, in terms of $\Delta M=M_{\pi^{+} \pi^{-} \mu^{+} \mu^{-}}-M_{\mu^{+} \mu^{-}}$. Data are from Ref. [31].

shape of a narrow Breit-Wigner resonance seems to be visible on the slope of the $\Upsilon\left(2{ }^{3} S_{1}\right)$ resonance, though with little more than $2 \sigma$ relevance. Nevertheless, by coincidence or not, it comes out exactly in the expected place, namely at $M_{\Upsilon\left(2^{3} S_{1}\right)}+38 \mathrm{MeV}$.

\subsection{Diphoton mass distributions}

We do not expect the $E(38) \rightarrow \gamma \gamma$ mode to be very large, since $E(38)$ couples to quarks proportionally to their masses, as we concluded above in connection with the observed oscillations. Moreover, diphoton data for the mass interval $10-100 \mathrm{MeV}$ are very rare and usually with low statistics.

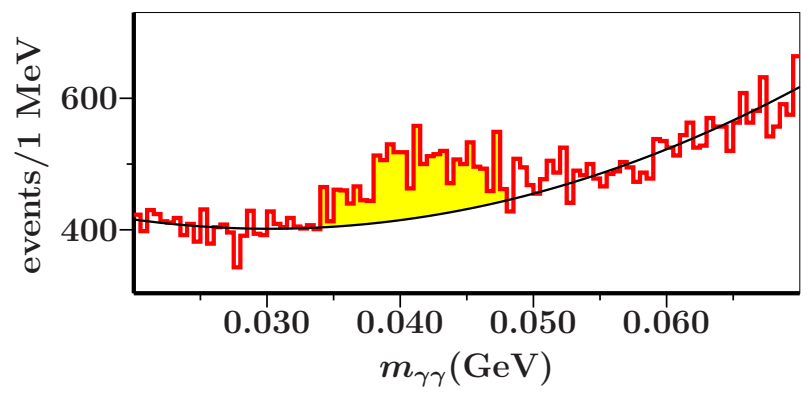

Figure 12. A modest signal in the $\gamma \gamma$ COMPASS [94] data around $40 \mathrm{MeV}$.

In Ref. [94], the COMPASS Collaboration studied $\omega$ and $\phi$ vector-meson production in $p p \rightarrow$ $p p \omega / \phi$ data, obtained at the two-stage magnetic COMPASS spectrometer attached to the SPS accelerator facility at CERN. For the indentification of the $\omega$ meson, the $\pi^{0}$ was reconstructed from two 
photons. A detail of the thus obtained invariant-mass distribution for $\gamma \gamma$ pairs is shown in Fig. 12, in which an enhancement at about $40 \mathrm{MeV}$ can be observed.

These data seem to have enough statistics to support the existence of a light boson with mass around $40 \mathrm{MeV}$. However, in a more recent version [94] of their work, the COMPASS Collaboration added the following remark to the figure caption regarding the enhancement in Fig. 15:

"The structures below the $\pi^{0}$ mass peak are artefacts of low energetic photon reconstruction due to secondary interactions in the detector material and to cuts in the reconstruction algorithm. They should not be mistaken for any physical signal."

Although it may of course be possible to obtain resonance-like structures by artefacts in data collection, we are convinced this is not the case for the signal in the $40 \mathrm{MeV}$ region, because it coincides surprisingly well with the other observations reported in Refs. [74, 86].

Furthermore, it is clear that the light boson cannot be an ordinary hadron emerging from a hadronic vertex, unless at an extremely low rate. Otherwise, it would have been observed long ago. Ordinary hadronisation in high-energy collisions gives rise to pions, kaons, and other hadrons that are stable with respect to strong interactions. These are processes in which quark-pair creation and gluonic jets dominate. But on the other hand, judging from the amount of events in the low-mass enhancement in the two-photon data, which is about $10 \%$ of the number of events in the $\eta$ signal, it does seem to be produced with a reasonably large rate in the COMPASS experiment. Such a rate indicates that it most probably is a hadronic particle, though with very peculiar properties that still have to be understood.

Fortunately, the COMPASS Collaboration also presented a Monte-Carlo (MC) simulation of the data in Ref. [95]. The authors of Ref. [95] gave the following list of mechanisms that may result in structures in their data.

- Secondary $\pi^{0}$ mesons produced in the detector material downstream of the target lead to diphoton masses which are below the nominal $\pi^{0}$ mass when reconstructed assuming a target vertex.

- Material concentrated in detector groups leads to peak-like structures.

- Secondary $e^{+} e^{-}$pairs from photon conversion in the spectrometer material lead to low-mass structures.

- Cuts applied in the reconstruction software lead to additional structure in the low-mass range.

Most of those processes occur, of course, in the EM calorimeter ECAL2, because it is further downstream than ECAL1, and therefore picks up more contamination due to unwanted processes.

These artefacts are reproduced in the MC simulation of Ref. [95] for the reactions under study, using a complete description of the spectrometer material and employing the same reconstruction software as for the real data analysis.

\subsection{Simulation of diphoton data}

In principle, the diphoton invariant-mass distribution below the nominal $\pi^{0}$ mass has a structure as represented by the solid curve in Fig. 13. However, the two electromagnetic calorimeters, ECAL1 at about 6 meters from the target and ECAL2 at about 30 meters downstream, do not accept low-energy photons. This results in the non-observation of the enhancement at zero invariant diphoton mass. Hence, at very low masses no events are observed, since they are removed by the trigger system of the EM calorimeters, as indicated by the removed area in Fig. 13. It has the effect that at low mass a peak shows up in the data. 


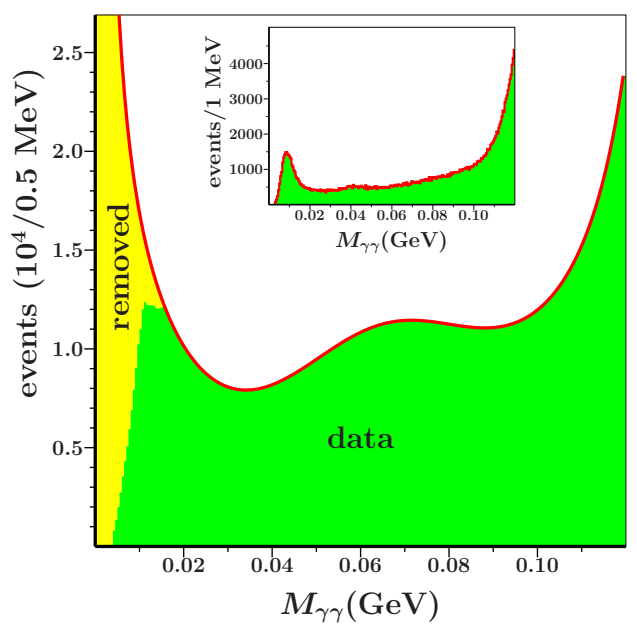

Figure 13. Invariant two-photon mass distributions below the nominal $\pi^{0}$ mass. The solid curve in principle indicates the general aspect of such a distribution. Data removed by the trigger system are represented by the removed area. So the data area in principle represents the final data. In the inset, we display the diphoton mass distribution for the $p p$ data of Ref. [94].

Now, the data selection system does of course also influence the aspect of the data for higher diphoton masses. It may even result in some structures which resemble resonances. Moreover, several physical processes in the experimental setup, not related to the purpose of the experiment, may result in further structures. In Fig. 14 we depict the MC simulation of Ref. [95]. We find that the

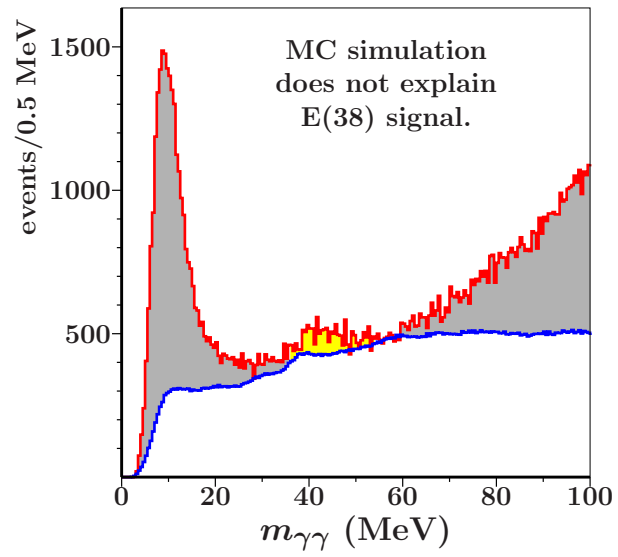

Figure 14. Diphoton mass distribution [94] (upper curve) and Monte-Carlo simulation [95] (lower curve).

experimental data are not at all well described by the MC simulation of Ref. [95]. The discrepancy between data and simulation at low diphoton masses is rather serious and may indicate differences between the experimental low-energy cuts and those of the simulation. We observe from Fig. 14 that for some reason the $\pi^{0}$ signal, which in the experimental data sets out at about $50 \mathrm{MeV}$, is not included in the simulations [95]. From the discrepancy between the actual $\pi^{0}$ tail and its simulation we may conclude that the spreading in the data may not have been correctly taken into account in the simulation. The latter observation would imply that the small effect of the artefacts in the present simulation should be even smaller. We may safely conclude that the MC simulation of Ref. [95] does not explain the signal around $40 \mathrm{MeV}$ [96]. 


\subsection{High-statistics signal}

In Ref. [97], the COMPASS Collaboration carried out a partial-wave analysis of $p \pi^{-} \rightarrow p \pi^{-} \eta^{\prime}$ in order to extract the exotic $J^{P C}=1^{-+} \pi^{-} \eta^{\prime} P$-wave signal. The analysis consists of various intermediate steps. The first step is to select $\pi^{0} \eta$ pairs, possibly stemming from the decay of an $\eta^{\prime}$-meson. Either one of the two mesons $\pi^{0}, \eta$ or both may decay into pairs of photons. Hence, as a byproduct of their analysis, the COMPASS Collaboration produced an invariant-mass distribution of photon pairs. In Fig. 15 we show a detail of the invariant two-photon mass distribution of COMPASS. These data

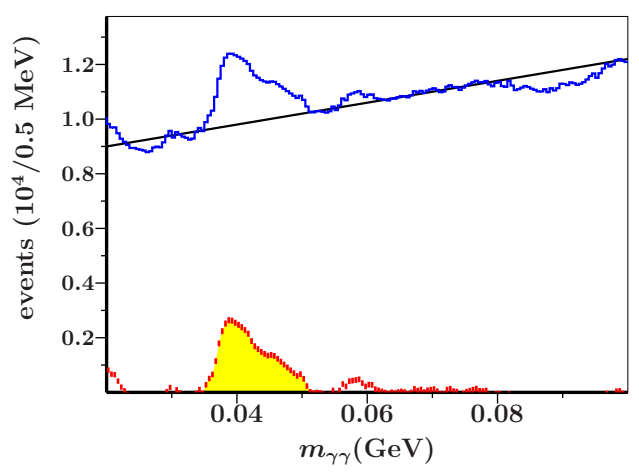

Figure 15. Top: a clear signal in the $\gamma \gamma$ COMPASS [97] data, with maximum at about $39 \mathrm{MeV}$. Bottom: the $E(38)$ structure that remains after background subtraction and contains about 46000 events.

seem to have enough statistics to substantiate the existence of a light boson with mass around $40 \mathrm{MeV}$.

\section{Conclusions}

With respect to the hadronic sector of the Standard Model, we have shown that high-statistics experiments are necessary for serious data analysis, in particular for the study of threshold enhancements. Moreover, we have exhibited data that may indicate the existence of a weak substructure.

The question whether there exists a (scalar) boson with a mass of about $38 \mathrm{MeV}$ does not depend exclusively on the existence of a resonance-like structure in the experimental data of Ref. [97], yet it is at present the clearest signal we have found in many experiments. Diphoton data for the mass interval $10-100 \mathrm{MeV}$ are very rare and usually with low statistics. Therefore, it is very important that the present issue be settled, which requires a profound understanding of all possible sources of artefacts. In this respect, we welcome the efforts of our colleagues at JINR (Dubna), who are reportedly finishing the analysis of their $E(38)$ signal [98].

With this latest piece of evidence of $E(38)$, we conclude that it is now necessary to establish its mass and other properties by further experiments. We think that $E(38)$ is the light scalar Higgstype boson which was proposed in a model describing the unification of electromagnetic and strong interactions [75]. Finally, as $E(38)$ appears to couple to quarks proportionally to their masses, its coupling to the top quark is expected to be quite strong.

\section{Acknowledgments}

This work was supported in part by the Fundação para a Ciência e a Tecnologia of the Ministério da Ciência, Tecnologia e Ensino Superior of Portugal, under contract CERN/FP/ 123576/2011. One of us (EvB) wishes to thank the organisers of the 3rd International Conference on New Frontiers in Physics for kindly inviting him to Kolymbari (Crete, Greece) and for the hospitality at the Conference Center of the Orthodox Academy of Crete. 


\section{References}

[1] G. Zweig, CERN Reports TH-401 and TH-412 (1963), see also Developments in the Quark Theory of Hadrons, Vol. 1, 22-101 (1981), edited by D. B. Lichtenberg and S. P. Rosen.

[2] M. Gell-Mann, Phys. Lett. 8, 214 (1964).

[3] H. Fritzsch, M. Gell-Mann and H. Leutwyler, Phys. Lett. B 47, 365 (1973).

[4] J. J. Aubert et al. [E598 Collaboration], Phys. Rev. Lett. 33, 1404 (1974).

[5] J. E. Augustin et al. [SLAC-SP-017 Collaboration], Phys. Rev. Lett. 33, 1406 (1974).

[6] P. A. Rapidis et al. [MARK-I Collaboration], Phys. Rev. Lett. 39, 526 (1977) [Erratum-ibid. 39, 974 (1977)].

[7] W. Bacino et al. [DELCO Collaboration], Phys. Rev. Lett. 40, 671 (1978).

[8] G. S. Abrams et al., Phys. Rev. D 21, 2716 (1980).

[9] R. Brandelik et al. [DASP Collaboration], Desy report 78/18, Phys. Lett. B 76, 361 (1978).

[10] J. Siegrist et al., Phys. Rev. Lett. 36, 700 (1976).

[11] S. W. Herb, D. C. Hom, L. M. Lederman, J. C. Sens, H. D. Snyder, J. K. Yoh, J. A. Appel and B. C. Brown et al., Phys. Rev. Lett. 39, 252 (1977).

[12] H. Albrecht et al. [ARGUS Collaboration], Phys. Lett. B 116, 383 (1982).

[13] K. G. Wilson, Phys. Rev. D 10, 2445 (1974).

[14] E. van Beveren and G. Rupp, Int. J. Theor. Phys. Group Theor. Nonlin. Opt. 11, 179 (2006) [arXiv:hep-ph/0304105].

[15] E. van Beveren, C. Dullemond, and G. Rupp, Phys. Rev. D 21, 772 (1980) [Erratum-ibid. D 22, 787 (1980)].

[16] E. van Beveren, G. Rupp, T. A. Rijken, and C. Dullemond, Phys. Rev. D 27, 1527 (1983).

[17] S. Godfrey and N. Isgur, Phys. Rev. D 32, 189 (1985).

[18] R. L. Jaffe, Phys. Rev. D 15, 267 (1977).

[19] John D. Weinstein and N. Isgur, Phys. Rev. D 41, 2236 (1990).

[20] E. van Beveren, T. A. Rijken, K. Metzger, C. Dullemond, G. Rupp and J. E. Ribeiro, Z. Phys. C 30, 615 (1986) [arXiv:0710.4067].

[21] D. Mohler, C. B. Lang, L. Leskovec, S. Prelovsek and R. M. Woloshyn, Phys. Rev. Lett. 111, 222001 (2013) [arXiv:1308.3175].

[22] E. van Beveren and G. Rupp, Phys. Rev. Lett. 91, 012003 (2003) [arXiv:hep-ph/0305035].

[23] C. B. Lang, L. Leskovec, D. Mohler and S. Prelovsek, Phys. Rev. D 86, 054508 (2012) [arXiv:1207.3204 [hep-lat]].

[24] P. Estabrooks, Phys. Rev. D 19, 2678 (1979).

[25] D. Aston et al. [LASS Collaboration], Nucl. Phys. B 296, 493 (1988).

[26] E. van Beveren and G. Rupp, Ann. Phys. 323, 1215 (2008) [arXiv:0706.4119].

[27] E. van Beveren and G. Rupp, Europhys. Lett. 81, 61002 (2008) [arXiv:0710.5823].

[28] E. van Beveren and G. Rupp, Europhys. Lett. 84, 51002 (2008).

[29] B. Aubert [BaBar Collaboration], Phys. Rev. Lett. 102, 012001 (2009) [arXiv:0809.4120].

[30] E. van Beveren and G. Rupp, Phys. Rev. D 80, 074001 (2009) [arXiv:0908.0242].

[31] B. Aubert et al. [BaBar Collaboration], Phys. Rev. D 78, 112002 (2008) [arXiv:0807.2014].

[32] E. van Beveren and G. Rupp, arXiv:1009.4097.

[33] M. Ablikim et al. [BES Collaboration], arXiv:0807.0494.

[34] K. A. Olive et al. [Particle Data Group Collaboration], Chin. Phys. C 38, 090001 (2014).

[35] S. Dubynskiy and M. B. Voloshin, Phys. Rev. D 78, 116014 (2008) [arXiv:0809.3780]. 
[36] X. Liu, Z. G. Luo, Y. R. Liu and S. L. Zhu, arXiv:0808.0073.

[37] B. Aubert [BaBar Collaboration], Phys. Rev. D 79, 092001 (2009) [arXiv:0903.1597].

[38] B. Aubert [BaBar Collaboration], Phys. Rev. Lett. 97, 222001 (2006) [arXiv:hep-ex/0607082].

[39] E. van Beveren and G. Rupp, Phys. Rev. Lett. 97, 202001 (2006) [arXiv:hep-ph/0606110].

[40] F. E. Close, C. E. Thomas, O. Lakhina, and E. S. Swanson, Phys. Lett. B 647, 159 (2007) [arXiv:hep-ph/0608139].

[41] B. Zhang, X. Liu, W. Z. Deng, and S. L. Zhu, Eur. Phys. J. C 50, 617 (2007) [arXiv:hep$\mathrm{ph} / 0609013]$.

[42] P. Colangelo, F. De Fazio and S. Nicotri, Phys. Lett. B 642, 48 (2006) [arXiv:hep-ph/0607245].

[43] F. De Fazio, Nucl. Phys. Proc. Suppl. 186, 363 (2009) [arXiv:0810.3549].

[44] A. Zhang, Nucl. Phys. A 856, 88 (2011) [arXiv:0904.2453].

[45] B. Aubert [BaBar Collaboration], Phys. Rev. D 80, 092003 (2009) [arXiv:0908.0806].

[46] E. van Beveren and G. Rupp, Phys. Rev. D 81, 118101 (2010) [arXiv:0908.1142].

[47] R. Aaij et al. [ LHCb Collaboration], Phys. Rev. D 90, 072003 (2014) [arXiv:1407.7712].

[48] R. Aaij et al. [LHCb Collaboration], arXiv:1407.7574 [hep-ex].

[49] E. van Beveren and G. Rupp, PoS HQL2010:003 (2010) [arXiv:1011.2360].

[50] S. Chatrchyan et al. [CMS Collaboration], Phys. Rev. Lett. 110, 081803 (2013) [arXiv:1212.6639].

[51] G. Aad et al. [ATLAS Collaboration], Phys. Lett. B 726, 120 (2012) [arXiv:1307.1432].

[52] G. Aad et al. [ATLAS Collaboration], Phys. Lett. B 716, 1 (2012) [arXiv:1207.7214].

[53] S. Chatrchyan et al. [CMS Collaboration], Phys. Lett. B 716, 30 (2012) [arXiv:1207.7235].

[54] V. Khachatryan et al. [ CMS Collaboration], Eur. Phys. J. C 74, 3076 (2014) [arXiv:1407.0558].

[55] S. M. Consonni [ for the ATLAS Collaboration], arXiv:1305.3315.

[56] U. Baur, H. Fritzsch and H. Faissner, Phys. Lett. B 135, 313 (1984).

[57] M. Leurer, H. Harari and R. Barbieri, Phys. Lett. B 141, 455 (1984).

[58] S. N. Biswas, S. Rai Choudhury, K. Datta and A. Goyal, Pramana 23, 607 (1984).

[59] F. Boudjema, Phys. Rev. D 36, 969 (1987).

[60] S. Narison and M. Perrottet, Nuovo Cim. A 90, 49 (1985).

[61] M. Yasue, Phys. Rev. D 39, 3458 (1989).

[62] H. Aihara et al. [TPC/TWO GAMMA Collaboration], Phys. Rev. Lett. 57, 3245 (1986).

[63] T. Matsushima, arXiv:1207.4387.

[64] E. Eichten, K. Lane and A. Martin, arXiv:1210.5462.

[65] H. Fritzsch, arXiv:1307.6400.

[66] E. H. Simmons, A. Atre, R. S. Chivukula, P. Ittisamai, N. Vignaroli, A. Farzinnia and R. Foadi, contribution to SCGT12 "KMI-GCOE workshop on strong coupling gauge theories in the LHC perspective", 4-7 Dec. 2012, Nagoya University, arXiv:1304.0255 [hep-ph].

[67] A. Belyaev, M. S. Brown, R. Foadi and M. T. Frandsen Phys. Rev. D 90, 035012 (2014) [arXiv:1309.2097].

[68] [CMS Collaboration], CMS-PAS-HIG-13-016.

[69] S. Chatrchyan et al. [ CMS Collaboration], Phys. Rev. D 89, 092007 (2014) [arXiv:1312.5353].

[70] M. Acciarri et al. [L3 Collaboration], Phys. Lett. B 479, 101 (2000) [arXiv:hep-ex/0002034].

[71] M. Acciarri et al. [L3 Collaboration], Phys. Lett. B 345, 609 (1995).

[72] [CMS Collaboration], CMS-PAS-HIG-13-001. March, 21 (2013).

[73] E. van Beveren, S. Coito and G. Rupp, arXiv:1304.7711. 
[74] E. van Beveren and G. Rupp, arXiv:1202.1739.

[75] C. Dullemond, T. A. Rijken and E. van Beveren, Nuovo Cim. A 80, 401 (1984).

[76] E. van Beveren, T. A. Rijken and C. Dullemond, Nijmegen-report THEF-NYM-79-11 (1979).

[77] E. van Beveren, C. Dullemond and T. A. Rijken, Phys. Rev. D 30, 1103 (1984).

[78] E. van Beveren, T. A. Rijken, C. Dullemond and G. Rupp, Lect. Notes Phys. 211, 331 (1984).

[79] H. Weyl, Annalen der Physik 364, 101 (1919).

[80] M. Dohse, arXiv:0706.1887.

[81] E. van Beveren and G. Rupp, published in the Proceedings of Meson-Nucleon Physics and the Structure of the Nucleon (MENU2010), May 31 - June 4, 2010, College of William and Mary, Williamsburg Virginia, (USA) AIP Conf. Proc. 1374, 421 (2011) [arXiv:1009.1778].

[82] E. van Beveren and G. Rupp, Chin. Phys. C 35, 319 (2011) [arXiv:1004.4368].

[83] E. van Beveren, and G. Rupp, Ann. Phys. 324, 1620 (2009) [arXiv:0809.1149].

[84] E. van Beveren and G. Rupp, published in Proc. Workshop Recent Developments in Particle and Nuclear Physics, April 30, 2001, Coimbra (Portugal), (Universidade de Coimbra, 2003) ISBN 972-95630-3-9, pages 1-16, [arXiv:hep-ph/0201006].

[85] E. van Beveren, D. V. Bugg, F. Kleefeld and G. Rupp, Phys. Lett. B 641, 265 (2006) [arXiv:hep$\mathrm{ph} / 0606022]$.

[86] E. van Beveren and G. Rupp, arXiv:1102.1863.

[87] E. van Beveren and G. Rupp, Phys. Rev. D 79, 111501R (2009) [arXiv:0905.1595].

[88] B. Aubert [BaBar Collaboration], Contributed to the 34th International Conference on High Energy Physics (ICHEP 2008), Philadelphia, Pennsylvania, 30 Jul - 5 Aug 2008, SLAC-PUB13360, BABAR-CONF-08-004, arXiv:0808.1543.

[89] E. van Beveren and G. Rupp, arXiv:1009.5191.

[90] V. M. Aulchenko et al. [CMD-2 Collaboration], JETP Lett. 82, 743 (2005) [Pisma Zh. Eksp. Teor. Fiz. 82, 841 (2005)] [arXiv:hep-ex/0603021].

[91] T. Aaltonen et al. [CDF Collaboration], Phys. Rev. Lett. 103, 152001 (2009) [arXiv:0906.5218].

[92] P. del Amo Sanchez et al. [BaBar Collaboration], Phys. Rev. Lett. 104, 191801 (2010) [arXiv:1002.4358].

[93] E. Guido [BaBar Collaboration], to appear in the proceedings of Meeting of the Division of Particles and Fields of the American Physical Society (DPF 2009), Detroit, Michigan, 26-31 Jul 2009, arXiv:0910.0423.

[94] J. Bernhard and K. Schönning [COMPASS Collaboration], eConf C110613, 146 (2011) [arXiv:1109.0272].

[95] J. Bernhard, J. M. Friedrich, T. Schlüter, K. Schönning [COMPASS Collaboration], arXiv:1204.2349.

[96] E. van Beveren and G. Rupp, arXiv:1204.3287.

[97] T. Schlüter [COMPASS Collaboration], eConf C110613, 302 (2011) [arXiv:1108.6191].

[98] Kh. U. Abraamyan, A. B. Anisimov, M. I. Baznat, K. K. Gudima, M. A. Nazarenko, S. G. Reznikov and A. S. Sorin, arXiv:1208.3829. 